\title{
Relationships between thalamostriatal neurons and pedunculopontine projections to the thalamus: a neuroanatomical tract-tracing study in the rat
}

Received: 7 December 1998 / Accepted: 8 March 1999

\begin{abstract}
The present study aimed to investigate whether the pedunculopontine projection to the thalamus overlaps with identified thalamostriatal neurons. These projections were studied using a dual tract-tracing procedure combining anterogradely transported biotinylated dextran amine (pedunculopontine projections) and retrogradely transported Fluoro-Gold (thalamostriatal projections). Overlapping thalamic territories between thalamostriatal neurons and the axon terminals arising from the pedunculopontine tegmental nucleus were observed in the midline (paraventricular) and in the intralaminar (centrolateral, central medial, paracentral and parafascicular) thalamic nuclei. Other thalamic nuclei, such as the ethmoid, intermediodorsal, mediodorsal, paratenial, posteromedian, ventromedian, ventrolateral and rhomboid thalamic nuclei, displayed a lesser degree of overlap. These observations suggest the existence of presumptive contacts between thalamostriatal neurons and axons emerging from the pedunculopontine tegmental nucleus, therefore supporting the possible existence of feedback circuits in the rat basal ganglia in which the tegmentothalamic projection would play a major role.
\end{abstract}

Key words Basal ganglia $\cdot$ Pedunculopontine tegmental nucleus $\cdot$ Fluoro-Gold $\cdot$ BDA

Abbreviations aca Anterior commissure, anterior part . $A c b$ accumbens nucleus - $a c p$ anterior commissure, posterior part - $A D$ anterodorsal thalamic nucleus . $A P T D$ anterior pretectal nucleus, dorsal part .

$A q$ aqueduct (Sylvius) $\cdot B$ basal nucleus (Meynert) $c c$ corpus callosum $\cdot C L$ centrolateral thalamic nucleus . $C M$ central medial thalamic nucleus $\cdot C n F$ cuneiform nucleus $\cdot C P u$ caudate putamen $\cdot E C I C$ external cortex of the inferior colliculus $\cdot E P$ entopeduncular nucleus .

E. Erro · J.L. Lanciego · J.M. Giménez-Amaya ( Departamento de Anatomía, Facultad de Medicina, Universidad de Navarra, C/Irunlarrea,

S/N, E-31080 Pamplona, Spain

e-mail:jmga@unav.es

Tel.: +34-48-42-56-85, Fax: +34-48-42-56-49
Eth ethmoid thalamic nucleus $F$ nucleus of the fields of Forel $\cdot f r$ fasciculus retroflexus $\cdot G P$ globus pallidus . IAM interanteromedial thalamic nucleus .

$I M D$ intermediodorsal thalamic nucleus $\cdot L D$ laterodorsal thalamic nucleus $\cdot L H b$ lateral habenular nucleus . $L P$ lateral posterior thalamic nucleus $\cdot L V$ lateral ventricle $\cdot M D$ mediodorsal thalamic nucleus . $M H b$ medial habenular nucleus $\cdot \mathrm{MiTg}$ microcellular tegmental nucleus $\cdot m l$ medial lemniscus . $m t$ mammillothalamic tract $\cdot P C$ paracentral thalamic nucleus $\cdot P F$ parafascicular thalamic nucleus .

$P o$ posterior thalamic nuclear group .

$P P T g$ pedunculopontine tegmental nucleus . $P V$ paraventricular thalamic nucleus $\cdot R e$ reuniens thalamic nucleus $\cdot R h$ rhomboid thalamic nucleus . $R t$ reticular thalamic nucleus $\cdot s c p$ superior cerebellar peduncle $\cdot s m$ stria medullaris of the thalamus . $S N$ substantia nigra $\cdot S P F$ subparafascicular thalamic nucleus $\cdot S P T g$ subpeduncular tegmental nucleus . $S T h$ subthalamic nucleus $\cdot S u b$ submedius thalamic nucleus $\cdot x s c p$ decussation of the superior cerebellar peduncle $\cdot V L$ ventrolateral thalamic nucleus . $V M$ ventromedial thalamic nucleus $V P M$ ventral posteromedial thalamic nucleus $\cdot V P L$ ventral posterolateral thalamic nucleus $\cdot V P P C$ ventral posterior thalamic nucleus, parvicellular part $\cdot Z I$ zona incerta

\section{Introduction}

The basal ganglia are a group of subcortical structures related to the organization of movement as well as to cognitive and motivational processes (Albin et al. 1995; Graybiel 1995; Parent and Hazrati 1995; Gerfen and Wilson 1996). The main output of the basal ganglia is directed to the cortex with a relay in the thalamus. The most caudal brain nucleus receiving projections from the basal ganglia is the pedunculopontine tegmental nucleus (PPTg). Anterograde and retrograde tract-tracing procedures and neurophysiological studies have reported the existence of a close and reciprocal relationship between 
Fig. 1 Camera lucida drawings of the injection sites in the $\mathrm{CPu}$ and in the PPTg corresponding to the cases included in this study. Schematic drawings are rostrocaudally arranged according to the injection site in the $\mathrm{CPu}$. The shading around some injection sites represents the spread of the tracer
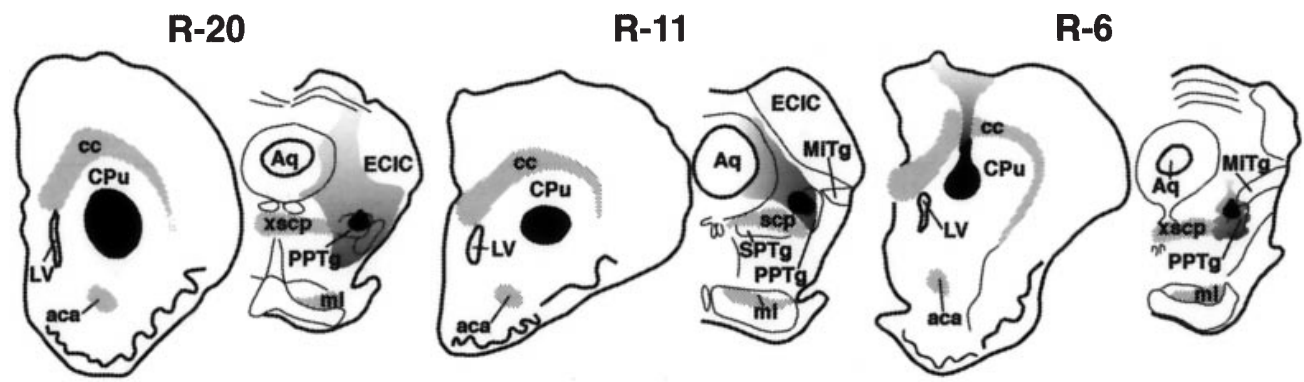

R-53
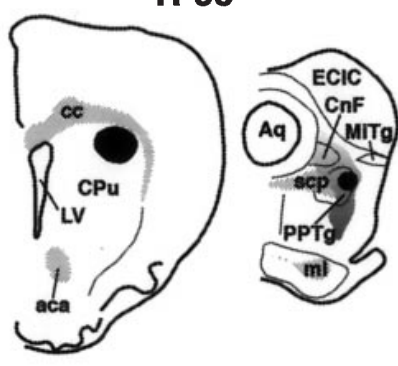

R-8

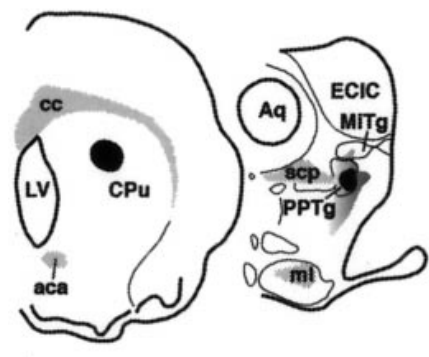

R-24

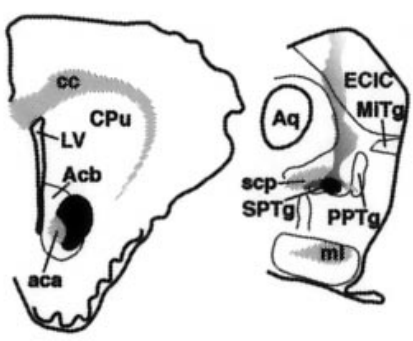

R-34

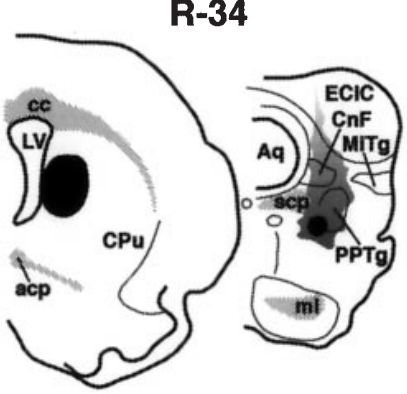

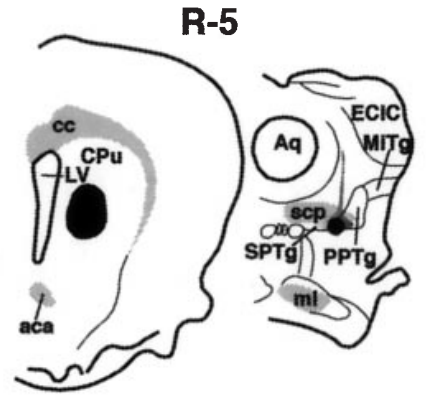

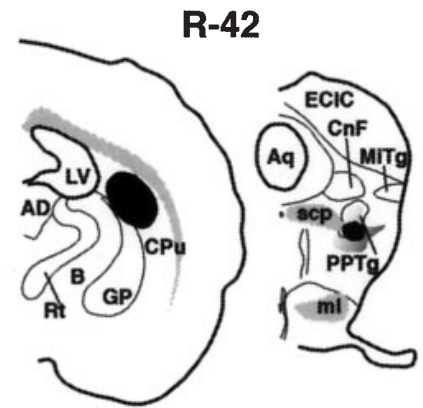

the PPTg and several basal ganglia structures such as the entopeduncular nucleus (EP), the subthalamic nucleus (STh) and the substantia nigra (SN) (Saper and Loewy 1982; Jackson and Crossman 1983; Scarnati et al. 1987a; Rye et al. 1987; Spann and Grofova 1989; Lavoie et al. 1990; Lavoie and Parent 1994a). Basal ganglia structures lack direct access to pontomedullary or spinal nuclei and the PPTg has therefore been considered a link between the basal ganglia and the brainstem and spinal cord. Furthermore, recent behavioral experiments have made it possible to hypothesize that the PPTg could be a critical site through which limbic information from the ventral striatum concerned with motivation and reinforcement can gain access to motor outflow coming from the dorsal striatum and directed towards pontomedullary systems (Winn et al. 1997). In addition to its connections with the basal ganglia, the PPTg sends a dense projection to the thalamus, which is implicated in the regulation of the sleep-wake cycle (Jones 1991).

As a part of our ongoing studies on the thalamic interaction between the input and output pathways of the basal ganglia and their related nuclei, we have examined

the relationship between the thalamostriatal neurons and the pedunculopontine projections to the thalamus, to elucidate the possible existence of thalamic areas of overlap between both pathways. Retrograde and anterograde tracer experiments with Fluoro-Gold (FG) and biotinylated dextran amine (BDA) were performed in the rat in order to identify the cells of origin of the thalamostriatal projections and the thalamic afferents coming from the PPTg and to further analyze the thalamic overlap of these projections in a single section.

\section{Materials and methods}

Female Wistar rats ( $n=9$; body weight ranging from 200 to $230 \mathrm{~g}$ ) were used in this study. Animals were anesthetized $(0.1 \mathrm{ml} / 100 \mathrm{~g})$ with an intramuscular injected mixture of $4: 3$ parts Ketaset (1\% of a solution of ketamine) and Rompun ( $2 \%$ of a solution of xylacine) respectively, and placed in a stereotaxic frame. In a single surgical session a $10 \%$ solution of biotinylated dextran amine (BDA, Molecular Probes Europe, Leiden, The Netherlands) in $0.01 \mathrm{M}$ phosphate buffer, $\mathrm{pH} 7.25$, was iontophoretically delivered into the PPTg through a glass micropipette (inner tip diameter $20-30 \mu \mathrm{m}$ ) using a $5 \mu \mathrm{A}$ positive pulsed direct current (7 s on/off 
Fig. 2A-D Camera lucida drawings of four coronal sections through the thalamus rostrocaudally arranged, showing the retrogradely labeled neurons and the anterogradely labeled fibers corresponding to case R-20. Inset illustrates the injection sites related to this case
A

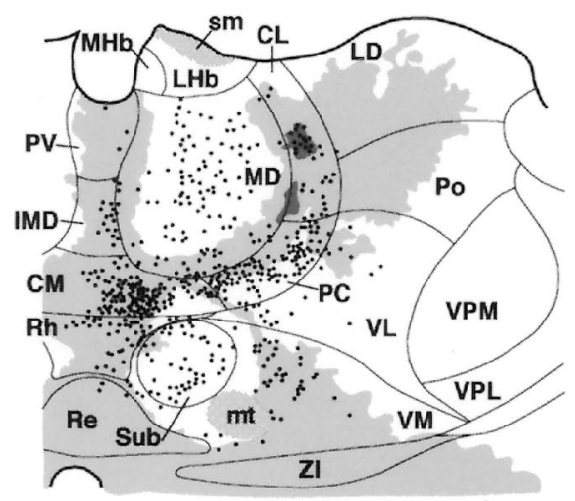

C

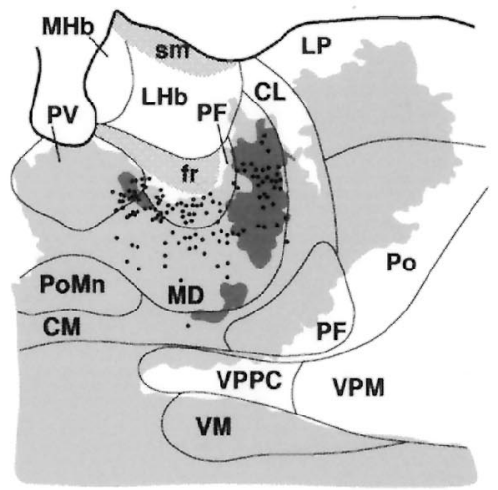

B

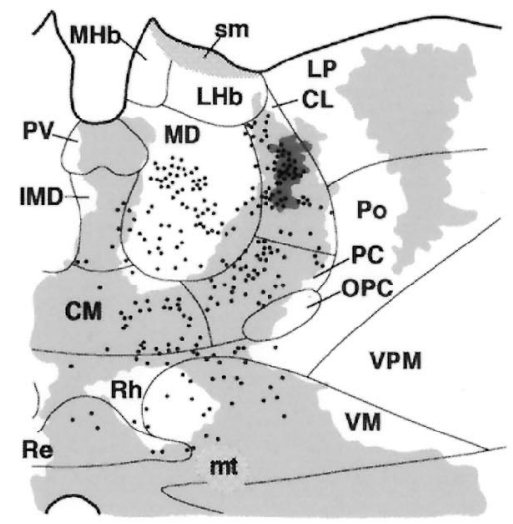

D

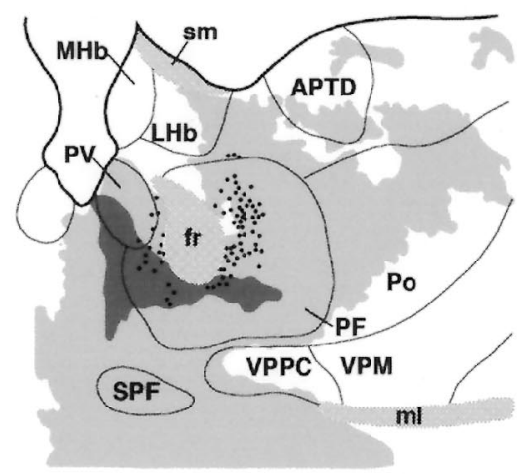

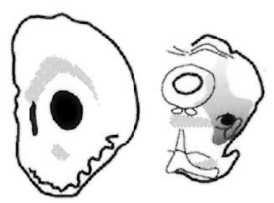

- FG labeled neurons

Low to moderate density of BDA labeled fibers

High density of BDA labeled fibers for $5 \mathrm{~min}$ ). Then, a $2 \%$ solution of Fluoro-Gold (FG, Fluorochrome, Englewood, CO) in 0.1 M cacodylate buffer, pH 7.3, was iontophoretically injected into the ipsilateral caudate putamen $(\mathrm{CPu})$, according to the same iontophoretical parameters described above for the BDA injections. Stereotaxic coordinates were taken from the atlas of Paxinos and Watson (1998). Animals were handled at all times according to the Society for Neuroscience Policy on the Use of Animals in Neuroscience Research and also the European Communities Council Directive 86/609/EEC. After a survival time of 7 days, the animals were anesthetized with an intraperitoneal overdose of $10 \%$ chloral hydrate in distilled water and perfused transcardially. Perfusion was performed with a saline-rinsing solution at body temperature, immediately followed by $500 \mathrm{ml}$ cold fixative containing $4 \%$ paraformaldehyde, $0.1 \%$ glutaraldehyde and $0.2 \%$ saturated picric acid in $0.125 \mathrm{M}$ phosphate buffer, $\mathrm{pH}$ 7.4. After the perfusion, the skull was opened and the brain removed and stored in a cryoprotective solution prepared according to the method of Rosene et al. (1986). Coronal frozen sections (40 $\mu \mathrm{m}$ thick) were obtained in a sliding microtome and collected in $0.125 \mathrm{M}$ phosphate buffer, $\mathrm{pH}$ 7.4. A total of ten series of each brain were obtained. The first series was processed for the simultaneous visualization of anterogradely transported BDA and somatopetally transported FG. Sections were first incubated in an $\mathrm{ABC}$ solution (avidin biotin complex, Vector, Burlingame, $\mathrm{CA}$ ) for $90 \mathrm{~min}$ and then reacted with $30 \% \mathrm{H}_{2} \mathrm{O}_{2}$ in the presence of nickel-enhanced diaminobenzidine solution (DAB-Ni) for 3-5 min. The incubation was continued in 1:2000 rabbit anti-FG (Chemicon, Temecula, LA) for $60 \mathrm{~h}$ at $40 \mathrm{C}$ under gentle agitation. Subsequently, sections were incubated in 1:50 swine anti-rabbit $\mathrm{IgG}$ for $120 \mathrm{~min}$ at room temperature and then in 1:600 rabbit-PAP (DAKO) for $90 \mathrm{~min}$ and finally stained with diaminobenzidine (DAB) (Sigma Chemical Co., St. Louis, MO) as the chromogen for the peroxidase reaction for 5-10 min. Extensive washing with $0.05 \mathrm{M}$ Tris-buffered saline at $\mathrm{pH} 8.0$ (TBS) with $0.5 \%$ Triton $\mathrm{X}$ 100 (TBS-Tx) was carried out throughout the procedure. Several rinsing steps with $0.05 \mathrm{M}$ Tris $\mathrm{HCl}, \mathrm{pH}$ 7.6, were also conducted prior to and after the incubations in the chromogen solutions. The first antiserum was prepared in TBS-Tx, $\mathrm{pH} 8.0$, plus $0.2 \%$ bovine serum albumin (BSA) (Merck, Darmstadt, Germany) to prevent non-specific binding. Once the staining was completed, the sections were mounted on glass slides using a $2 \%$ solution of gelatin in $0.05 \mathrm{M}$ Tris $/ \mathrm{HCl}, \mathrm{pH} 7.6$, dried, quickly dehydrated in toluene, and coverslipped with Entellan. Adjacent sections were processed for acetylcholinesterase (AChE) and Nissl stains.

The exact location of the injection sites, the retrogradely labeled neurons and the anterogradely labeled fibers in the thalamus were directly plotted using a camera lucida. Each drawing displaying labeling was then scanned (Arcus II, AGFA) in a computer (Power Macintosh 9500/150) and both the thalamic contours and the structures displaying labeling were redrawn using Freehand 
Fig. 3A-D Camera lucida drawings of four coronal sections through the thalamus rostrocaudally arranged, showing the retrogradely labeled neurons and the anterogradely labeled fibers corresponding to case R-24. Inset illustrates the injection sites related to this case
A

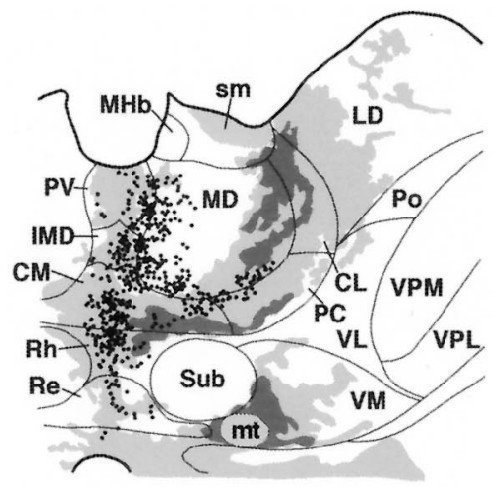

C
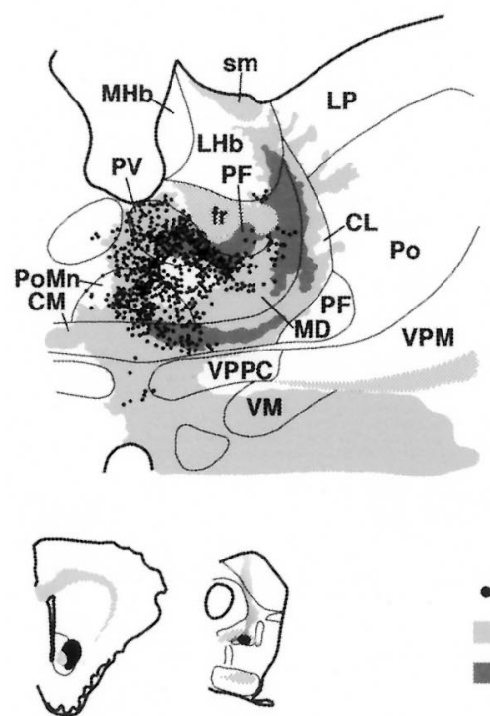

B

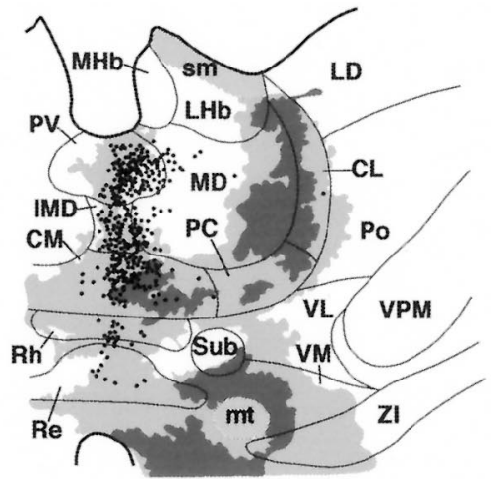

D

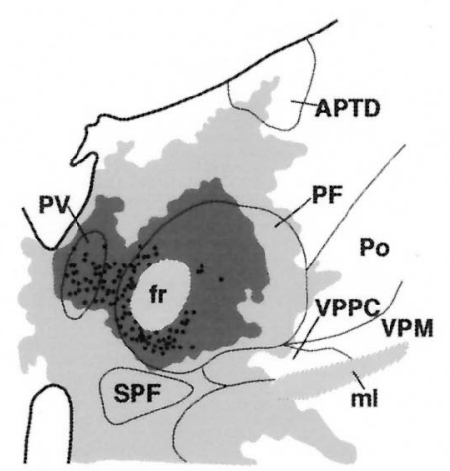

5.5.1 (Macromedia Inc., 1988-1995). In order to better delineate the different thalamic nuclei for the accurate location of both the thalamostriatal neurons and the thalamic pedunculopontine afferents, the adjacent Nissl- and AChE-stained series were used as a reference for the cytoarchitecture.

\section{Results}

\section{Injection sites}

The retrograde tracer FG was delivered in the striatum at different stereotaxic coordinates in an effort to cover a wide extent of this nucleus. One injection site was placed in the accumbens nucleus (Acb) (R-24). The anterograde tracer BDA was injected at different rostrocaudal levels of the PPTg. Furthermore, two cases (R-5 and $\mathrm{R}-34)$ received a BDA injection in the border between the subpeduncular tegmental nucleus (SPTg) and the PPTg, while the BDA injection in case R-24 was placed in the SPTg itself. Leakage of the tracer along the injection track, resulting in a slight cortical contamination, was only noticed in one case (R-6). Nevertheless, no significant differences regarding the number and distribution of the thalamic labeled neurons were observed. Figure 1 summarizes the location and extent of the tracer deposits in the present study.

\section{Distribution of retrogradely labeled neurons} in the thalamus

After deposits of FG within the $\mathrm{CPu}$, retrogradely labeled neurons were always found in the ipsilateral midline and intralaminar thalamic nuclei irrespective of the location of the FG injection. Retrogradely labeled neurons were found in the ventral, lateral and posterior thalamic groups as a function of the rostrocaudal location of the injection sites into the $\mathrm{CPu}$. Dorsal $\mathrm{CPu}$ injections re- 

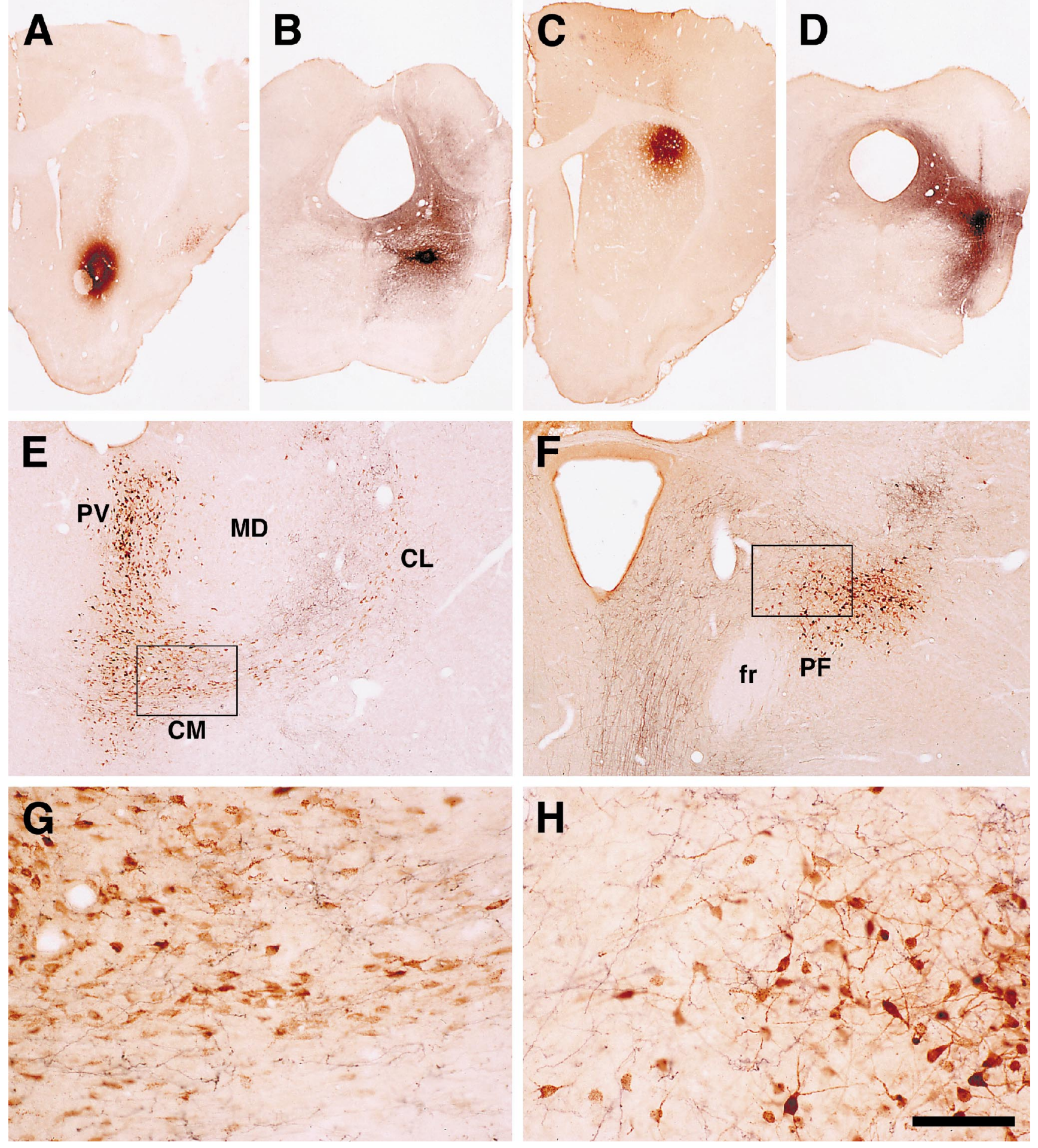

Fig. 4 A, B Injection sites of case R-24. C, D Injection sites of case R-53. E Low-power photomicrograph of a coronal section through the thalamus showing the thalamostriatal neurons and the pedunculopontine fibers in the CM thalamic nucleus of case R-24. F Low-power photomicrograph showing the thalamostriatal neurons and the pedunculopontine fibers in the PF thalamic nucleus of case R-53. G Inset taken from E. H Inset taken from F. Scale bar $2000 \mu \mathrm{m}(\mathbf{A}-\mathbf{D}) ; 500 \mu \mathrm{m}(\mathbf{E}, \mathbf{F}) ; 100 \mu \mathrm{m}(\mathbf{G}, \mathbf{H})$

sulted in a remarkable amount of neuronal labeling in the following thalamic nuclei: centrolateral $(\mathrm{CL})$, central medial (CM), paracentral (PC) and parafascicular (PF) thalamic nuclei (Fig. 4F). Moderate to low numbers of neuronal labeling were observed in the ethmoid (Eth), mediodorsal (MD), paratenial (PT), ventrolateral (VL) and ventromedial (VM) thalamic nuclei (Fig. 2). The FG injection in the Acb resulted in abundant neuronal labeling in the $\mathrm{CM}$ and paraventricular (PV) thalamic nuclei 
and more scarcely in the interanteromedial (IAM), intermediodorsal (IMD), MD, reuniens (Re), rhomboid (Rh), $\mathrm{PC}, \mathrm{PF}$ and posteromedian (PoMn) thalamic nuclei (Figs. 3, 4E). Thalamic neuronal labeling located in the ventral nuclei displayed the following differences regarding the rostrocaudal axis: rostral $\mathrm{CPu}$ injections elicited a preferential neuronal labeling in the VM and VL nuclei, while after caudal FG deposits in the $\mathrm{CPu}$, neuronal labeling was mainly found in the ventral posterior, lateral and posterior thalamic groups.

\section{Distribution of anterogradely labeled fibers}

After restricted BDA injections within the PPTg, labeled fibers were abundantly found in the basal forebrain (substantia innominata, preoptic area, nuclei of the stria terminalis and septal nuclei), hypothalamus, pretectal area and superior colliculus. Different degrees of labeling were found in basal ganglia structures: high to moderate anterograde labeling was constantly found in the ventral pallidum (VP), EP, STh and SN. Moderate to low anterograde labeling was found in the $\mathrm{CPu}$ and in the Acb. BDA terminal fields were found in several thalamic nuclei, with a dominant distribution within the midline and intralaminar nuclei (Figs. 2, 3). Lower to moderate densities of anterogradely labeled fibers were found in the VM, VL, VPM-VPL, LD, LP and Po thalamic nuclei (Figs. 2, 3). Almost no differences in the distribution of labeling related to the location of the tracer deposits in the PPTg were noticed. The density of the BDA thalamic labeling varies slightly as a function of the location of the injection sites in the PPTg. The strongest densities were observed with rostral BDA deposits, while weaker projections were noticed in the animals receiving caudal BDA injections. Tracer deposits in the SPTg (R-24) also displayed strong thalamic labeling (Fig. 3).

Thalamic areas of overlap between retrograde and anterograde labeling

Thalamic areas displaying overlap between the terminal fibers arising from the PPTg to the thalamus and thalamostriatal neurons are summarized in Fig. 5. The thalamic area of constant and the most intense degree of convergence between both projections irrespective of the FG injection sites was the PF thalamic nucleus (Figs. $2 \mathrm{D}, 3 \mathrm{D}, 4 \mathrm{~F}, \mathrm{H})$. A constant overlap was also found in the $\mathrm{CM}$ and Rh thalamic nuclei (Figs. 2A,B, 3A,B, 4E,G). In most cases, the CL, MD, PC and PV thalamic nuclei also presented areas of overlap between these projections (Figs. 2A-C, 3A-C). Inconstant and a lesser degree of overlap was found in the Eth, IMD, PT, PoMn, VM and VL thalamic nuclei.

The distribution of retrogradely labeled neurons within the thalamus is determined by the location of the tracer deposit into the $\mathrm{CPu}$. The thalamic distribution of anterogradely labeled fibers is constant independently of

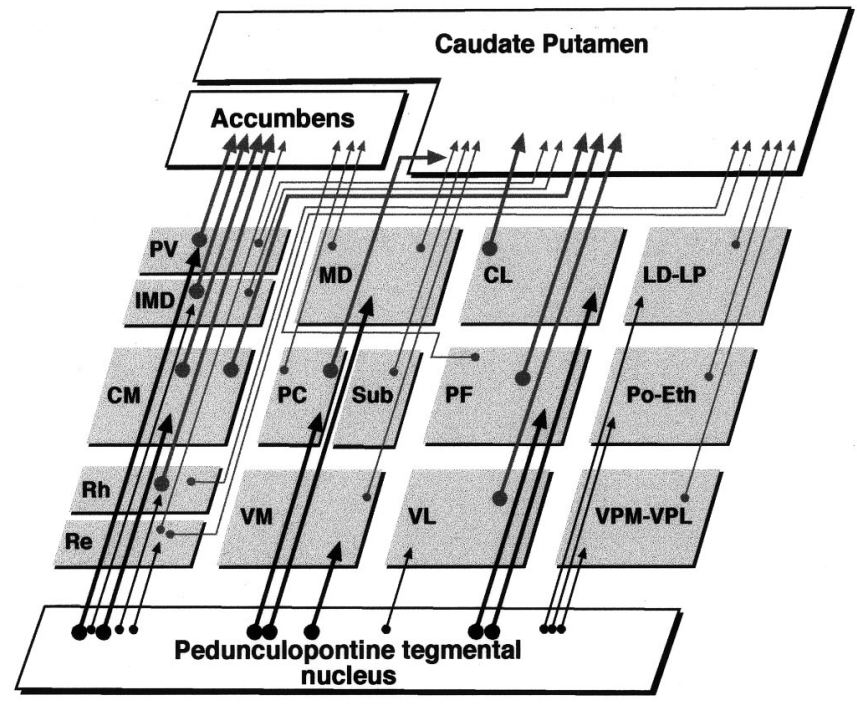

Fig. 5 Schematic diagram summarizing the general pattern of the thalamostriatal projection to the $\mathrm{CPu}$ and to the Acb and the projections from the PPTg to the different thalamic nuclei. The strength of the projections is illustrated by the different line thicknesses. The PPTg projects to all the different thalamic nuclei with the exception of the submedius thalamic nucleus $(S u b)$. The midline and intralaminar CL, CM, PC, PF and PV thalamic nuclei, as well as the MD and VM, receive dense projections. The Acb receives projections mainly from the midline nucleus while the $\mathrm{CPu}$ receives projections from the intralaminar thalamic nuclei and less often from other thalamic nuclei

the location of the BDA injection within the PPTg. Therefore, the degree and distribution of overlap between these two projection systems seem to depend only on the placement of the $\mathrm{CPu}$ injections. After FG injections in the Acb, the thalamic nuclei showing most intense overlap were the CM, PF and PV (Fig. 3), while after injections in the dorsal and lateral $\mathrm{CPu}$, the highest degree of overlap was found in the CL, lateral MD and PF thalamic nuclei (Fig. 2).

\section{Discussion}

\section{Technical considerations}

The reliability of the anterograde and retrograde tracttracing procedures used in this study has been widely documented elsewhere (Schmued and Heimer 1990; Veeman et al. 1992; Rajakumar et al. 1993). A serious drawback to injecting a retrograde tracer within the $\mathrm{CPu}$ is the possible uptake of the tracer by the thalamocortical fibers. This possibility seems unlikely in our procedure because the FG injections pass through a very small area of the cerebral cortex which, according to its size, only receives very sparse projections. Therefore, we consider that the retrogradely labeled thalamic neurons observed in this study originate from terminals located in the $\mathrm{CPu}$, rather than from cortical terminals. Another point of concern is the possible uptake of the retrograde tracer by fibers of passage, as the $\mathrm{CPu}$ of the rat is traversed by nu- 
merous small bundles of cortifugal and corticopetal fibers. Although some discrepancy remains regarding the FG uptake by fibers of passage (Schmued and Fallon 1986; Pieribone and Aston-Jones 1988; Dado et al. 1990; Divac and Mogensen 1990), there is general agreement as to the reduced amount of transport of FG by damaged fibers traversing the injection site. This uptake is kept to a minimum by means of iontophoretic delivery of the dye (Pieribone and Aston-Jones 1988; Schmued and Heimer 1990). Moreover, complementary data from experiments in our laboratory with anterograde tracer injections in the thalamus have shown a distribution of terminal fields in the $\mathrm{CPu}$, supporting the present results regarding the thalamostriatal projection.

\section{Thalamic territories of overlap}

The present study provides evidence of the existence of thalamic areas of overlap between thalamostriatal labeled neurons and axon terminals emerging from the PPTg. Thalamostriatal labeled neurons were predominantly found in midline and intralaminar nuclei. These findings are in agreement with earlier anatomical studies in the rat (Van der Kooy 1979; Veening et al. 1980; Berendse and Groenewegen 1990). We have found a lateral to medial gradient of neuronal labeling in the thalamic nuclei corresponding to a dorsal to ventral gradient of the injection sites in the $\mathrm{CPu}$, as previously reported (Berendse and Groenewegen 1990).

Retrograde and anterograde tract-tracing studies have demonstrated in the rat that PPTg cholinergic neurons project directly to the midline and intralaminar thalamic nuclei as well as to other thalamic nuclei (Sugimoto and Hattori 1984; Sofroniew et al. 1985; Hallanger et al. 1987, 1990; Grunwerg et al. 1992). The distribution of BDA anterogradely labeled fibers in the thalamus and other subcortical structures that we have found in this study is in agreement with previous reports (Hallanger and Wainer 1988).

The reciprocal connections existing between the PPTg and the basal ganglia have been extensively studied (Saper and Loewy 1982; Jackson and Crossman 1983; Rye et al. 1987; Lee et al. 1988), with emphasis on their relationship with the SN (Beninato and Spencer 1987; Clarke et al. 1987; Scarnati et al. 1987b; Beninato and Spencer 1988; Gould et al. 1989; Spann and Grofova 1991; Lavoie and Parent 1994). The pedunculonigral projection may be an indirect way of modulating striatal activity, as PPTg nigral afferents synapse onto dopaminergic nigrostriatal neurons (Tokuno et al. 1988; Bolam et al. 1991; Hernández-López et al. 1992). In our study, a PPTg projection has been abundantly found in the SN, $\mathrm{STh}$, ET and VP, and only moderately within the $\mathrm{CPu}$ and Acb. The relationship existing between PPTg projections to the basal ganglia and the PPTg projection to the thalamus has not yet been clarified (Scarnati et al. 1987a). It is interesting to note that the information conveyed to the thalamus from the PPTg could influence the basal ganglia by the direct thalamostriatal projection as well as by the indirect thalamocorticostriatal pathway (Dubé et al. 1988; Inglis and Winn 1995).

Based on its projections to the pontine, medullar and spinal structures, the PPTg has been considered a possible link between the striatum and lower brainstem sites (Inglis and Winn 1995). The description of a triad projection from a single PPTg neuron to the thalamus, brainstem and SN supports the role of the PPTg as an integrative structure that could receive different information and then connect simultaneously with different systems (Takakusaki et al. 1996). These recent findings have allowed the hypothesis that the PPTg is a critical site where motor outflow from the dorsal striatum and limbic information from the ventral striatum converge and can gain access to pontomedullary nuclei (Winn et al. 1997).

The major finding of this study is the existence of several thalamic nuclei in which thalamostriatal neurons and axon terminals arising from the PPTg appear to overlap. The thalamic nuclei with a higher degree of convergence seem to be the CL, CM, MD, PF and PV. Therefore, the present study could support the existence of a feedback circuit between the basal ganglia and the PPTg through the thalamus. It is interesting to note that overlapping territories between thalamostriatal cells and nigrothalamic projections have also been recently described in the cat (Heras et al. 1997, 1998) as well as in the primate (McFarland et al. 1998). Our findings are highly suggestive of presumptive contacts between PPTg fibers and thalamostriatal neurons, although ultrastructural confirmation will be required. However, two issues remain to be elucidated. First, we do not know whether or not PPTg projections to thalamostriatal neurons are cholinergic. Ultrastructural observations in the dog indicate that at least some thalamostriatal neurons are innervated by cholinergic terminals which probably arise from the PPTg (Isaacson and Tanaka 1988). Second, we also do not know whether they are collateral fibers of those connecting with thalamocortical neurons. Although there has been some controversy regarding this point (Steriade and Glenn 1982; Royce 1983; Macchi et al. 1984; Cesaro et al. 1985), there is a growing body of evidence that suggests that thalamostriatal neurons send collaterals to the cortex. Anterograde and retrograde studies in the rat have found some neurons in the PF, and PV thalamic nuclei send collaterals to the cortex as well as to various components of the basal ganglia (Deschênes et al. 1996; Otake and Nakamura 1998). In both thalamic nuclei we have found marked areas of overlap between thalamostriatal neurons and PPTg afferent fibers. Considering that thalamocortical neurons of the midline and intralaminar thalamic nuclei are under the influence of cholinergic inputs coming from the PPTg (Jones 1991; Hallanger et al. 1990), and bearing in mind that in those thalamic nuclei most thalamocortical neurons send collaterals to the striatum, it can be speculated that the ascending PPTg projection to the thalamus may modulate cortical and basal ganglia activity simultaneously. 
Acknowledgements Supported by FIS 96/0488, Fundacioń "Marcelino Botín" and Universidad de Navarra.

\section{References}

Albin RL, Young AB, Penney JB (1995) The functional anatomy of disorders of the basal ganglia. Trends Neurosci 18:63-64

Beninato M, Spencer RF (1987) A cholinergic projection to the rat substantia nigra from the pedunculopontine tegmental nucleus. Brain Res 412:169-174

Beninato M, Spencer RF (1988) The cholinergic innervation of the rat substantia nigra: a light and electron microscopic immunohistochemical study. Exp Brain Res 72:178-184

Berendse HW, Groenewegen HJ (1990) Organization of thalamostriatal projection in the rat, with special emphasis on the ventral striatum. J Comp Neurol 299:187-228

Bolam JP, Francis CM, Henderson Z (1991) Cholinergic input to dopaminergic neurons in the substantia nigra: a double immunocytochemical study. Neuroscience 41:483-494

Cesaro P, Nguyen-Legros J, Pollin B, Laplane S (1985) Single intralaminar thalamic neurons project to cerebral cortex, striatum and nucleus reticularis thalami. A retrograde anatomical study in the rat. Brain Res 325:29-37

Clarke PBS, Hommer DW, Pert A, Skirboll LR (1987) Innervation of substantia nigra neurons by cholinergic afferents from pedunculopontine nucleus in the rat: neuroanatomical and electrophysiological evidence. Neuroscience 23:1011-1019

Dado RJ, Burstein R, Cliffer KD, Giesler GJ Jr (1990) Evidence that Fluoro-Gold can be transported avidly through fibers of passage. Brain Res 553:329-333

Deschênes M, Bourassa J, Doan VD, Parent A (1996) A single cell study of the axonal projections arising from the posterior intralaminar thalamic nuclei in the rat. Eur J Neurosci 8:329-343

Divac I, Mogensen J (1990) Long-term retrograde labelling neurons. Brain Res 524:339-341

Dubé L, Smith AD, Bolam JP (1988) Identification of synaptic terminals of thalamic or cortical origin in contact with distinct medium-size spiny neurons in the rat neostriatum. J Comp Neurol 267:455-471

Gerfen CR, Wilson CJ (1996) The basal ganglia. In: Swanson LW, Björklund A, Hökfelt T (eds) Handbook of chemical neuroanatomy, vol 12: integrated systems of the CNS, part III. Elsevier, Amsterdam, pp 371-468

Gould E, Woolf NJ, Butcher LL (1989) Cholinergic projections to the substantia nigra from the pedunculopontine and laterodorsal tegmental nuclei. Neuroscience 28:611-623

Graybiel AM (1995) The basal ganglia. Trends Neurosci 18:60-62

Grunwerg BS, Krein H, Krauthamer GM (1992) Somatosensory input and thalamic projection of pedunculopontine tegmental neurons. Neuroreport 3:673-675

Hallanger AE, Wainer BH (1988) Ascending projections from the pedunculopontine tegmental nucleus and adjacent mesopontine tegmentum in the rat. J Comp Neurol 274:483-515

Hallanger AE, Levey AI, Lee HJ, Rye DB, Wainer BH (1987) The origins of cholinergic and other subcortical afferents to the thalamus in the rat. J Comp Neurol 262:105-124

Hallanger AE, Price SD, Lee HJ, Steininger TL, Wainer BH (1990) Ultrastructure of cholinergic synaptic terminals in the thalamic anteroventral, ventroposterior and dorsal lateral geniculate nuclei of the rat. J Comp Neurol 299:482-492

Heras S de las, Mengual E, Velayos JL, Giménez-Amaya JM (1997) New data on the anatomical organization of the thalamostriatal projections. Adv Neurol 74:69-81

Heras S de las, Mengual E, Giménez-Amaya JM (1998) Overlapping territories between the thalamostriatal and nigrothalamic projections in cats. Neuroreport 9:1913-1916

Hernández-López S, Góngora-Alfaro JL, Martínez-Fong D, Aceves J (1992) A cholinergic input to the substantia nigra pars compacta increases striatal dopamine metabolism by in vivo voltammetry. Brain Res 598:114-120
Inglis WL, Winn P (1995) The pedunculopontine tegmental nucleus: where the striatum meets the reticular formation. Prog Neurobiol 47:1-29

Isaacson LG, Tanaka JRD (1988) Cholinergic innervation of canine thalamostriatal projection neurons: an ultrastructural study combining choline acetyltransferase immunocytochemistry and WGA-HRP retrograde labeling. J Comp Neurol 277: 529-540

Jackson A, Crossman AR (1983) Nucleus tegmenti pedunculopontinus: efferent connections with special reference to the basal ganglia, studied in the rat by anterograde and retrograde transport of horseradish peroxidase. Neuroscience 10:725-765

Jones BE (1991) Paradoxical sleep and its chemical/structural substrates in the brain. Neuroscience 40:637-656

Lavoie B, Parent A (1994a) Pedunculopontine tegmental nucleus in the squirrel monkey: projections to the basal ganglia as revealed by anterograde tract-tracing methods. J Comp Neurol 344:210-231

Lavoie B, Parent A (1994b) Pedunculopontine tegmental nucleus in the squirrel monkey: cholinergic and glutamatergic projections to the substantia nigra. J Comp Neurol 344:232-241

Lavoie B, Desjardins C, Parent A (1990) Projections from the pedunculopontine tegmental nucleus (PPN) to basal ganglia in primate. Soc Neurosci Abstr 16:236

Lee HJ, Rye DB, Hallanger AE, Levey AI, Wainer BH (1988) Cholinergic vs. noncholinergic efferents from the mesopontine tegmentum to the extrapyramidal motor system. J Comp Neurol 275:469-492

Macchi G, Bentivoglio M, Molinari M, Minciacchi D (1984) The thalamo-caudate versus thalamo-cortical projections as studied in the cat with fluorescent retrograde double labeling. Exp Brain Res 54:225-239

McFarland NR, Fudge JL, Haber SN (1998) An examination of the relationship between pallidal and nigral afferents and the VA/VL thalamostriatal projection. Soc Neurosci Abstr 24:662

Otake K, Nakamura Y (1998) Single midline thalamic neurons projecting to both the ventral striatum and the prefrontal cortex in the rat. Neuroscience 86:635-649

Parent A, Hazrati L-N (1995) Functional anatomy of the basal ganglia. I. The cortico-basal ganglia-thalamocortical loop. Brain Res Rev 20:91-127

Paxinos G, Watson C (1998) The rat brain in stereotaxic coordinates. Academic Press, Sydney

Pieribone VA, Aston-Jones G (1988) The iontophoretic application of Fluoro-Gold for the study of afferents to deep brain nuclei. Brain Res 475:259-271

Rajakumar N, Elisevich K, Flumerfelt BA (1993) Biotinylated dextran: a versatile anterograde and retrograde neuronal tracer. Brain Res 607:47-53

Rosene DL, Roy NJ, Davis BJ (1986) A cryoprotection method that facilitates cutting frozen sections of whole monkey brains for histological and histochemical processing without freezing artifact. J Histochem Cytochem 34:1301-1316

Royce GJ (1983) Single thalamic neurons which project to both the rostral cortex and caudate nucleus studied with the fluorescent method. Exp Neurol 79:773-784

Rye DB, Saper CB, Lee HJ, Wainer BH (1987) Pedunculopontine nucleus of the rat: cytoarchitecture, cytochemistry, and some extrapyramidal connections of the mesopontine tegmentum. J Comp Neurol 259:483-528

Saper CB, Loewy AD (1982) Projections of the pedundulopontine tegmental nucleus in the rat: evidence for an additional extrapyramidal circuitry. Brain Res 252:367-372

Scarnati E, Gasbarri A, Campana E, Pacitti C (1987a) The organization of nucleus tegmenti pedunculopontinus neurons projecting to basal ganglia and the thalamus: a retrograde fluorescent double labeling study in the rat. Neurosci Lett 79:1116

Scarnati E, Proia A, Di Loreto S, Pacitti C (1987b) The reciprocal electrophysiological influence between the nucleus tegmenti pedunculopontinus and the substantia nigra in normal and decorticated rats. Brain Res 423:116-124 
Schmued LC, Fallon JH (1986) Fluoro-Gold: a new fluorescent retrograde axonal tracer with numerous unique properties. Brain Res 377:147-154

Schmued LC, Heimer L (1990) Iontophoretic injection of fluorogold and other fluorescent tracers. J Histochem Cytochem 38:721-723

Sofroniew MV, Priestley JV, Consolazione A, Eckenstein F, Cuello AC (1985) Cholinergic projection from the midbrain and pons to the thalamus in the rat, identified by combined retrograde tracing and choline acetyltransferase immunohistochemistry. Brain Res 329:213-223

Spann BM, Grofova I (1989) Origin of ascending and spinal pathways from the nucleus tegmenti pedunculopontinus in the rat. J Comp Neurol 283:13-27

Spann BM, Grofova I (1991) Nigropedunculopontine projection in the rat: an anterograde tracing study with Phaseolus vulgarisleucoagglutinin (PHA-L). J Comp Neurol 311:375-388

Steriade M, Glenn LL (1982) Neocortical and caudate projections of intralaminar thalamic neurons and their synaptic excitation from midline reticular core. J Neurophysiol 48:352-371

Sugimoto T, Hattori T (1984) Organization and efferent projections of nucleus tegmenti pedunculopontinus pars compacta with special reference to its cholinergic aspects. Neuroscience $11: 931-946$
Takakusaki K, Shiroyama T, Yamamoto T, Kitai ST (1996) Cholinergic and non cholinergic tegmental pedunculopontine projection neurons in rats revealed by intracellular labeling. J Comp Neurol 371:345-361

Tokuno H, Moriizumi T, Kudo M, Nakamura Y (1988) A morphological evidence for monosynaptic projections from the nucleus tegmentipedunculopontinus pars compacta (TPC) to nigrostriatal projection neurons. Neurosci Lett 85:1-4

Van der Kooy D (1979) The organization of the thalamic, nigral and raphe cells projecting to the medial vs lateral caudateputamen in rat. A fluorescent retrograde double labeling study. Brain Res 169:381-387

Veenman CL, Reiner A, Honig MG (1992) Biotinylated dextran amine as an anterograde tracer for single and double label studies. J Neurosci Methods 41:239-254

Veening JG, Cornelissen FM, Lieven PAJM (1980) The topical organization of the afferents to the caudatoputamen of the rat. A horseradish peroxidase study. Neuroscience 5:1253-1268

Winn P, Brown VJ, Inglis WL (1997) On the relationships between the striatum and the pedunculopontine tegmental nucleus. Crit Rev Neurobiol 11:241-261 